SLAC-PUB-11285

SU-ITP-05/24

hep-th/0507069

\title{
An index for the Dirac operator on D3 branes with background fluxes
}

\author{
Eric Bergshoeff ${ }^{1}$, Renata Kallosh ${ }^{2,3}$, Amir-Kian Kashani-Poor ${ }^{2,4}$, \\ Dmitri Sorokin ${ }^{5}$, Alessandro Tomasiello ${ }^{2}$ \\ ${ }^{1}$ Centre for Theoretical Physics, University of Groningen, Nijenborgh 4, \\ 9747 AG Groningen, The Netherlands \\ 2 Department of Physics, Stanford University, Stanford, CA 94305-4060, USA. \\ ${ }^{3}$ Kyoto University, Yukawa Institute, Kyoto, 606-8502 JAPAN. \\ ${ }^{4}$ SLAC, Stanford University, Stanford, CA 94305-4060, USA. \\ ${ }^{5}$ INFN Sezione di Padova \& Dipartimento di Fisica "Galileo Galilei", \\ Università degli Studi di Padova, 35131, Padova, Italy
}

\begin{abstract}
We study the problem of instanton generated superpotentials in Calabi-Yau orientifold compactifications directly in type IIB string theory. To this end, we derive the Dirac equation on a Euclidean D3 brane in the presence of background fluxes. We propose an index which governs whether the generation of a superpotential in the effective $4 \mathrm{~d}$ theory by D3 brane instantons is possible. Applying the formalism to various classes of examples, including the $K 3 \times \frac{T^{2}}{\mathbb{Z}_{2}}$ orientifold, in the absence and presence of fluxes, we show that our results are consistent with conclusions attainable via duality from an M-theory analysis.
\end{abstract}

e-mails: E.A.Bergshoeff@rug.nl, kallosh@stanford.edu, kashani@slac.stanford.edu, dmitri.sorokin@pd.infn.it, tomasiel@stanford.edu 


\section{Introduction}

Stabilization of moduli is an important step on the path towards connecting $\mathrm{M} /$ string theory to effective particle physics and cosmology in $4 \mathrm{~d}$. Many interesting models with stabilization of all moduli have been discovered over the last few years. We refer the reader to the most recent paper on this topic [1, which contains a review of the current situation regarding stabilization of moduli in different versions of string theory and a detailed list of important references.

To obtain a realistic model, this stabilization should lead to $4 \mathrm{~d}$ de Sitter space with a tiny positive cosmological constant to describe the current acceleration of the universe. Deriving such effective phenomenological models from $\mathrm{M} /$ string theory presents a great challenge. The technical tools for such an enterprise are rather limited: in IIA string theory all moduli may be stabilized in anti de Sitter space using fluxes. The remaining issue is to uplift the cosmological constant to a positive value; for recent developments on this issue see [2]. The same problem of uplifting is still to be solved for heterotic string theory.

On the other hand, in type IIB theory the problem of uplifting has a reasonable solution. However, stabilization of all moduli in this theory cannot be achieved at the tree supergravity level, since fluxes stabilize only complex structure moduli and not Kähler moduli. Thus non-perturbative effects like gaugino condensation or instanton corrections due to Euclidean D3 branes wrapped on some 4-cycles of the compactified space should be taken into account. In [3] Witten studied instanton generated superpotentials in M-theory and F-theory compactifications on CY 4-folds. He derived a necessary constraint on the divisor the M5 instanton is wrapping in order for the generation of a superpotential to be possible. The conclusion was that it must have holomorphic characteristic $\chi=1$. Various aspects of this constraint and its possible modification due to fluxes have been studied in [4, 5, 6, 7. The papers [6, 7] use the Dirac operator on the M5 brane in the presence of fluxes [8]; counting the number of fermionic zero modes in the presence of fluxes and determining the corresponding flux-dependent index of the Dirac operator leads to a modification of the $\chi=1$ condition, giving rise to new possibilities for the stabilization of moduli.

In the interesting cosmological models [9] based on compactification on the $K 3 \times \frac{T^{2}}{\mathbb{Z}_{2}}$ orientifold with $\mathrm{N}=2$ supersymmetry [10, 11], the moduli of the compactification are distributed in vector and hypermultiplets. Moduli in vector multiplets, for example the volume of $K 3$, may be stabilized via gaugino condensation, since these moduli are the effective gauge coupling constants for some vector fields. For the moduli in hypermultiplets, no such terms are available, and one may hope for instanton generated superpotentials due to Euclidean D3 branes to achieve the desired stabilization. For example, the volume of the $\frac{T^{2}}{\mathbb{Z}_{2}}$ space is a modulus in a hypermultiplet [11] and cannot be stabilized by fluxes and/or gaugino condensation. Can it be stabilized by instanton corrections?

¿From the results in M-theory [6, 7] one may deduce via the connection to F-theory that in type IIB string theory compactified on $K 3 \times \frac{T^{2}}{\mathbb{Z}_{2}}$, an instanton generated superpotential is possible. In the presence of flux that is of type $(2,1)$ and primitive, the Euclidean D3 branes wrapped on $K 3$ as well as those on $\mathbb{P}^{1} \times \frac{T^{2}}{\mathbb{Z}_{2}}$ (where $\mathbb{P}^{1}$ is a projective plane within $K 3)$ can give rise to superpotentials. Thus, the stabilization of all Kähler moduli which were 
left unfixed by fluxes becomes possible.

The purpose of this paper is to initiate a systematic study of instanton corrections to the superpotential directly in type IIB string theory. This will allow us to draw conclusions on D3 brane instanton generated superpotentials without having to rely on M-theory/IIB duality. For earlier results on instanton corrections in type IIB flux backgrounds see [12, and also [13, where the problem is considered from the point of view of bosonic zero modes.

Our analysis deviates from complete generality in two points which we would like to point out here:

- The choice of bulk and worldvolume fluxes: We constrain our analysis in this paper to the case that the 2-form $\mathcal{F}=d A+B_{2}$ is absent on the Euclidean D3 brane, and we choose the background flux to be a primitive $(2,1)$-form. Both restrictions can be removed in a straightforward way and more general setting of the problem can be given, however this will lead to more complicated equations. This we postpone to future studies.

- Constant axion-dilaton: A generic IIB background including D7 branes will involve an axion-dilaton field which varies over the internal manifold. In section 5, we consider the simple situation for which this field is constant.

We should also note that in the study of some particular orientifold examples we encounter the following feature: the orientifold condition must be compatible with the choice of a gauge that fixes the $\kappa$-symmetry. Our choice, which is covariant under duality, is compatible with both the $K 3 \times \frac{T^{2}}{\mathbb{Z}_{2}}$ and $\frac{T^{6}}{\mathbb{Z}_{2}}$ compactifications, which we discuss in section [6] In each new example one should check that the relevant gauge-fixing condition is compatible with orientifolding.

The paper is organized as follows. We begin in section 2 by proposing an index for the Dirac operator on the D3 brane in the background of fluxes which, when different from 1, is to rule out instanton generated superpotentials. In section 3 , we comment on the connection between supersymmetries preserved by the background and fermionic zero modes on the D3 brane. In section 4, we derive the form of the Dirac operator on the D3 brane. In section 5 , we turn to solving the Dirac equation. The index proposed in section 2 passes its first test here; it predicts that before orientifolding and turning on fluxes, instantons do not generate a superpotential. In section [ 6 we consider the following orientifolds: $K 3 \times \frac{T^{2}}{\mathbb{Z}_{2}}$, more generally a Fano manifold, and $\frac{T^{6}}{\mathbb{Z}_{2}}$. We consider brane instantons wrapping several types of divisors in each of the cases, compute the corresponding index and find examples when a superpotential can be generated. In the discussion section, we explain the significance of adding space-filling D3 branes to our setup and raise the issue of their mobility. 


\section{An index for the Dirac operator on the D3 brane}

We wish to apply, following Witten [3], the analysis of the zero modes of the Dirac operator on a Euclidean D3 brane to the question of when such instantons can generate superpotentials in the effective 4d theory. Recall that in M-theory Witten identifies the charge of the M5 worldvolume fermions under $U(1)$ transformations in the normal bundle direction with the R-symmetry of the effective $3 \mathrm{~d}$ theory. By studying the zero modes of these fermions, he determines the transformation of the path integral measure under this normal bundle gauge group. Knowing that the full theory must be anomaly free (as was established in a series of subsequent papers [14, 15, 16]) allows him to deduce the effective transformation property of the instanton action under this normal bundle $U(1)$, from which he can conclude whether the exponentiated instanton action can play the role of a superpotential. We wish to perform the same analysis in the type IIB setup. Note that the anomaly structure of the D3 brane is much simpler than that of the M5 brane, as the worldvolume theory cannot have a gravitational anomaly. Witten's analysis however relies on the normal bundle anomaly. As the worldvolume fermions transform in a complex representation of the $\mathrm{SO}(2)$ normal bundle gauge group, this symmetry is also potentially anomalous for the type IIB D3 brane. We hence introduce the following index for the Dirac operator on the D3 brane,

$$
\chi_{D 3}=\frac{1}{2}\left(N_{+}-N_{-}\right),
$$

where $N_{ \pm}$is the number of fermionic zero modes with the $U(1)$ charge $\pm \frac{1}{2}$ in the normal direction.

In the presence of fluxes, the definition of the index must be modified, since the flux dependence of the Dirac operator can give rise to zero modes of mixed chirality. Taking into account that the flux must transform under normal bundle rotations allows us to assign a definite transformation property to these solutions as well (which was christened F-chirality in [6]).

Following the reasoning in [3], see also [6], we require $\chi_{D 3}=1$ for the generation of a superpotential by instantons to be possible.

\section{Broken supersymmetry and fermionic zero modes}

Here we will present a comment on our findings in [8] and [6] in a form which generalizes to the D3 brane. In gauge theories, it is well known that supersymmetries of the background which are broken by gauge instantons manifest themselves as zero modes of the charged fermions (one acts on a purely bosonic instanton solution with the supercharges; those supercharges that are conserved annihilate the solution, the broken supercharges generate fermionic solutions of the equations of motion, i.e. in particular of the Dirac equation). A similar result holds for brane instantons and zero modes of worldvolume fermions, as we will now argue for M5 branes. The equations presented in the next section will then demonstrate that the same arguments go through for D3 branes and are actually generic for all branes. 
The supersymmetries preserved by the background are solutions to the equation

$$
\delta \psi_{M} \epsilon=0,
$$

where $\delta \psi_{M} \epsilon$ is the supersymmetry transformation of the gravitino, i.e. $\delta_{\epsilon} \psi_{M}=\delta \psi_{M} \epsilon$ and $M$ is the $11 \mathrm{~d}$ space-time index. The supersymmetries preserved by the M5 brane are solutions to (3.1) which satisfy a further constraint [17, 18. In conventions ${ }^{1}$ in which the $\kappa$-symmetry of the brane is given by $\delta \theta=\left(1-\Gamma_{M 5}\right) \kappa$, this constraint takes the form

$$
\left(1+\Gamma_{M 5}\right) \epsilon=0 .
$$

Supersymmetries satisfying this constraint are preserved, because the transformation $\delta \theta=\epsilon$ can be undone by a $\kappa$-symmetry transformation in this case. The number of supersymmetries broken by the brane are hence

$$
\#\{\text { solutions to (3.1) }\}-\#\{\text { solutions to (3.2) }\} \text {. }
$$

The observation that allows us to relate this number to the number of zero modes of the worldvolume fermion is that the linear part of the fermionic equation on the M5 brane in the presence of background fluxes (but setting the field strength of the 2-form on the brane to zero) is given by

$$
\left(1-\Gamma_{M 5}\right) \Gamma^{\alpha} \delta \psi_{\alpha} \theta=0 .
$$

Here, $\alpha$ stands for M5 brane worldvolume indices, bulk quantities are pulled back onto the brane via $\delta \psi_{\alpha}=\delta \psi_{M} \partial_{\alpha} x^{M}$ and $\Gamma_{\alpha}=\Gamma_{\underline{M}} e^{\underline{M}}{ }_{M} \partial_{\alpha} x^{M}$, the index $M$ is the curved elevendimensional space-time index and $\underline{M}$ is the 'flat' tangent space index. We see that the pullback of each solution to (3.1) is a solution to the Dirac equation (3.4). Due to $\kappa$ symmetry, not all of these solutions correspond to dynamical fermions. The most natural gauge-fixing condition for this symmetry, which we shall call the duality covariant choice, ${ }^{2}$ is

$$
\frac{1}{2}\left(1-\Gamma_{M 5}\right) \theta=\theta_{-}=0, \quad \theta_{g . f .}=\theta_{+} \equiv \frac{1}{2}\left(1+\Gamma_{M 5}\right) \theta .
$$

Solutions to (3.4) which are not dynamical are those which are pure gauge, i.e. those that are annihilated by the $\kappa$-symmetry projector,

$$
\left(1+\Gamma_{M 5}\right) \theta=0,
$$

and which thus can be removed by the gauge fixing condition, like $\theta_{-}$in (3.5). The number of dynamical zero modes of the Dirac equation is hence

$$
\#\{\text { solutions to (3.4) }\}-\#\{\text { solutions to (3.6) }\} \text {. }
$$

\footnotetext{
${ }^{1}$ [17, 18] use the opposite sign conventions.

2 "Duality covariant" means that, as one can check, upon the double dimensional reduction the M5 brane $\kappa$-symmetry projector (and hence the gauge fixing condition) reduces to that for the D4 brane, which upon a chain of $\mathrm{T}$-dualities can be transformed into the $\kappa$-symmetry projectors of other Dp branes [19].
} 
Comparing (3.7) with (3.3), the claim that the number of zero modes of the worldvolume fermion is at least the number of supersymmetries of the background broken by the brane follows. But in addition to these zero modes, the Dirac equation $\gamma^{\alpha} \delta \psi_{\alpha} \epsilon=0$ may have more solutions than those of $\delta \psi_{M} \epsilon=0$. This is also familiar from gauge instantons: not all fermionic zero modes need to be related to supersymmetry.

The type of gauge (3.5) was introduced in [20, 18] where the structure of the Killing spinor of the supergravity background BPS configuration was used for fixing $\kappa$-symmetry. This gauge was used in [8, 6], where the above conditions were solved in terms of an irreducible $\operatorname{Spin}(1,5) \times \operatorname{Spin}(5)$ spinor of the M5 brane worldvolume, yielding the Dirac equation in gauge fixed form. In the general case of $\kappa$-symmetric superstrings, M2, M5 and Dp branes a similar gauge has been studied from the geometric perspective of superembedding [21, 22, 23, 24] ensuring that it is compatible with any supergravity background and with the corresponding gauge fixing of worldvolume diffeomorphisms.

\section{Dirac equation on D3 brane with background fluxes}

We now focus our attention on the D3 brane. In what follows we shall put to zero the DiracBorn-Infeld two-form flux $\mathcal{F}=d A+B_{2}$ on the D3 brane worldvolume. This is admissible for those compactified solutions of type IIB supergravity for which the pullback of the NS-NS form $B_{2}$ on the D3-brane worldvolume is pure gauge, i.e. $B_{2}=-\left.d A\right|_{D 3}$.

Our starting point is the quadratic Lagrangian for D3 brane fermions (without the DBI field contribution) derived in [19, 25] from an M2 brane Lagrangian by applying a chain of dualities $^{3}$

$$
L_{f}^{D 3}=\frac{1}{2} e^{-\phi} \sqrt{-\operatorname{det} g} \bar{\theta}\left(1-\Gamma_{D 3}\right)\left[\Gamma^{\alpha} \delta \psi_{\alpha}-\delta \lambda\right] \theta .
$$

The equations for unbroken supersymmetry of the background are

$$
\delta \psi_{m} \theta=0, \quad \delta \lambda \theta=0,
$$

(where $m$ stands for a curved 10d index), and the gauge fixing condition for the action of $\kappa$-symmetry $\delta_{\kappa} \theta=\left(1-\Gamma_{D 3}\right) \kappa$ reads

$$
\left(1-\Gamma_{D 3}\right) \theta=0,
$$

where $\Gamma_{D 3}=\sigma_{2} \gamma_{5}$, with $\sigma_{2}$ acting on a doublet of the Majorana-Weyl spinors $\theta=\left(\theta^{1}, \theta^{2}\right)$ and $\gamma_{5}$ a product of four ten-dimensional gamma matrices pulled back on the brane. Comparing to the previous section, we see that the arguments concerning the number of supersymmetries and fermionic zero modes presented there for the M5 brane apply equally well here.

The detailed form of the action (4.1) has in addition to the 'free' Dirac operator some torsion-type terms due to 3 -fluxes, which we denote by $T_{3}$ and some terms due to 1 -form

\footnotetext{
${ }^{3}$ A more economic way to get the Dirac Lagrangian for Dp branes (used for the M2 and M5 brane in [8]) would be to consider the linearized limit of the generic Dp brane fermionic equation first obtained in [22].
} 
and 5-form fluxes, which we denote by $T_{(1,5)}$. The action before gauge-fixing reads

$$
L_{f}^{D 3}=\frac{1}{2} e^{-\phi} \sqrt{-\operatorname{det} g} \bar{\theta}\left(1-\sigma_{2} \gamma_{5}\right)\left[\Gamma^{\alpha} \nabla_{\alpha}+T_{3}+T_{(1,5)}\right] \theta
$$

Here

$$
T_{3}=\frac{1}{8} H_{\alpha n p} \Gamma^{\alpha n p} \sigma_{3}-\frac{1}{24} H_{m n p} \Gamma^{m n p} \sigma_{3}+\frac{1}{8} e^{\phi} F_{\alpha n p}^{\prime} \Gamma^{\alpha n p} \sigma_{1}-\frac{1}{24} e^{\phi} F_{m n p}^{\prime} \Gamma^{m n p} \sigma_{1}
$$

and

$$
T_{(1,5)}=-\frac{1}{2} \Gamma^{m} \partial_{m} \phi+\frac{1}{4} e^{\phi} F_{\alpha} \Gamma^{\alpha}\left(i \sigma^{2}\right)+\frac{1}{8 \cdot 4 !} e^{\phi} F_{\alpha n p q r} \Gamma^{\alpha n p q r}\left(i \sigma^{2}\right)
$$

where $\alpha$ stands for the worldvolume directions of the D3 brane and the fluxes $H_{3}, F_{3}^{\prime}, F_{5}$ and $F_{m}$ are defined in the appendix. We basically use the notation and conventions of [25].

We next impose the gauge fixing condition (4.3), which is analogous to eq. (3.5). It can also be given in the form

$$
\theta_{2}=i \gamma_{5} \theta_{1}
$$

which implies that

$$
\theta=\left(\begin{array}{c}
\theta_{1} \\
i \gamma_{5} \theta_{1}
\end{array}\right), \quad \bar{\theta}=\left(\theta_{1}, i \theta_{1} \gamma_{5}\right)
$$

or

$$
\frac{1}{2}\left(1+\sigma_{2} \gamma_{5}\right) \theta=\theta, \quad \frac{1}{2}\left(1-\sigma_{2} \gamma_{5}\right) \theta=0
$$

We now push the projection operator $\frac{1}{2}\left(1-\sigma_{2} \gamma_{5}\right)$ in the Lagrangian through the gamma and Pauli matrices and let it act on $\theta$ which gives either $\theta$ back or zero depending on which of the two equations in (4.9) apply, which in turn is determined by whether the operator was commuted past flux with an even or odd number of legs in the D3-brane direction. We thus find the following expression for the Lagrangian

$$
\begin{aligned}
& L_{f}^{D 3}=\sqrt{-\operatorname{det} g} \theta_{1}\left\{2 e^{-\phi} \Gamma^{\alpha} \nabla_{\alpha}+\frac{1}{4} \tilde{G}_{\alpha \beta i} \Gamma^{\alpha \beta i}-\frac{1}{12} \tilde{G}_{i j k} \Gamma^{i j k}+\frac{i}{2 \cdot 4 !} \gamma_{5} F_{\alpha i j k l} \Gamma^{\alpha i j k l}+\frac{i}{2} \nabla_{\alpha} \tilde{\tau} \Gamma^{\alpha}\right\} \theta_{1} \\
& =\sqrt{-\operatorname{det} g} \theta_{1}\left\{2 e^{-\phi} \Gamma^{\alpha} \nabla_{\alpha}+\frac{1}{4} \tilde{G}_{\alpha \beta i} \Gamma^{\alpha \beta i}-\frac{1}{12} \tilde{G}_{i j k} \Gamma^{i j k}+\frac{i}{2 \cdot 4 !} \gamma_{5} F_{\alpha i j k l} \Gamma^{\alpha i j k l}+\frac{i}{2} \gamma_{5} \Gamma^{\alpha} \partial_{\alpha} C_{(0)}\right\} \theta_{1},
\end{aligned}
$$

where $i, j, k, l$ index the 6 directions orthogonal to the D3 brane, and

$$
\tilde{G}_{m n p} \equiv e^{-\phi} H_{m n p}+i F_{m n p}^{\prime} \gamma_{5}, \quad \tilde{\tau}=C_{(0)} \gamma_{5}+i e^{-\phi}
$$

Note that in the kappa-symmetry gauge under consideration the only $G_{3}$ flux components which appear in the Dirac Lagrangian are those which have one or three legs in the directions orthogonal to the D3 brane, while the contributions of the dilaton $\phi$ derivative vanishes. Note also that because of the self-duality of $F_{5}$ and $\Gamma^{m_{1} \cdots m_{5}}$ there is only the single term 
describing the coupling of the D3-brane fermions to the $F_{5}$ flux, which has one leg on the brane. Seemingly differente $F_{5}$ contributions which appear in the Lagrangian are related by duality as follows

$$
F_{\alpha i_{1} \cdots i_{4}} \Gamma^{\alpha i_{1} \cdots i_{4}}=-2 F_{\alpha \beta \gamma i j} \Gamma^{\alpha \beta \gamma i j} .
$$

By duality, the form of the D3 brane Dirac Lagrangian should be related to the M5 brane Dirac Lagrangian derived in 8]. Note also that in the chosen duality covariant gauge the Dirac Lagrangian (4.10) has a simpler form than in the gauge $\theta_{2}=0$ imposed in [26, 12, 25]. Moreover, as we shall show in section 6, it is the duality covariant gauge fixing condition which is compatible with the $K 3 \times \frac{T^{2}}{\mathbb{Z}_{2}}$ orientifold example which is of particular interest to us for its phenomenological applications.

To study instanton effects, we should now pass from Minkowski to Euclidean signature. In the Dirac Lagrangian (4.10) this will basically result in replacing $\sqrt{-\operatorname{det} g}$ with $\sqrt{\operatorname{det} g}$ and the Majorana-Weyl spinor $\theta_{1}$ with a complex Weyl spinor, since the Majorana condition is absent in $10 d$ Euclidean space. Note that though the Lagrangian becomes complex, the complex conjugate of $\theta_{1}$ never appears and hence, the number of the fermionic degrees of freedom over which the path integral is taken remains the same as in space-time of Minkowski signature, i.e. sixteen.

In what follows we shall consider fluxes

$$
G_{m n p} \equiv F_{m n p}-\tau H_{m n p}
$$

that have all legs in the compact $3 \mathrm{~d}$ complex space, and we will consider the axion-dilaton $\tau=C_{(0)}+i e^{-\phi}$ as a constant that is fixed by fluxes. Under these assumptions the Lagrangian (4.10) reduces to

$$
L_{f}^{D 3}=2 \sqrt{\operatorname{det} g} \theta_{1}\left\{e^{-\phi} \Gamma^{\alpha} \nabla_{\alpha}+\frac{1}{8} \tilde{G}_{\alpha \beta i} \Gamma^{\alpha \beta i}\right\} \theta_{1},
$$

where the index $i$ is now transverse to the brane and along the internal manifold. Note that eq. (4.13) coincides, up to an overall factor, with the D3 brane fermion Lagrangian calculated in the gauge $\theta_{2}=0$ in [12, for the case when all the fluxes except for $\tilde{G}_{\alpha \beta i}$ are zero (eq. (107) of that paper). When we consider the most general case of non-zero fluxes, the form of the Dirac Lagrangian in the duality covariant gauge (4.10) however is simpler and differs in some flux terms from the corresponding Lagrangians in the gauge $\theta_{2}=0$ (see e.g. eqs. (49), (51) and (56) of [12] and eq. (73) of [25] with the worldvolume flux set to zero). It would be of interest to understand this difference in detail, for instance from the perspective of brane dynamics and gauge fixing worldvolume diffeomorphisms which should be compatible with the $\kappa$-symmetry gauge choice.

\section{Determining the index}

We will perform the calculation of the index in two steps. We first solve the Dirac equation on the covering space of the orientifold and determine the number of zero modes with appropriate sign. We then impose the orientifold condition, which removes some of the modes from the spectrum. 


\subsection{Solving the Dirac equation}

The argument in section 2 regarding the possibility of generating a superpotential relies on the background having $\mathcal{N}=1$ unbroken supersymmetry in the effective $4 \mathrm{~d}$ theory. This means that we should consider only a $G_{3}$ flux which is $(2,1)$ and primitive, as shown in 29].

Let us review the setup. The D3 brane is wrapped on a 4-cycle $C$ inside the compactified 3-fold/orientifold. The six real dimensions of the internal complex 3 -fold include the directions tangent to the D3, $a, b, \bar{a}, \bar{b}$, and normal to it, $z, \bar{z}$. Rotations in $z, \bar{z}$ form the $S O(2) \sim U(1)$ symmetry whose anomaly is under consideration.

The worldvolume spinor has 10d chirality, so the zero modes will be of the form $\epsilon_{4}^{+} \otimes \epsilon_{6}^{+}$ and $\epsilon_{4}^{-} \otimes \epsilon_{6}^{-}$, with the superscripts indicating the $4 \mathrm{~d}$ and $6 \mathrm{~d}$ chirality, respectively. We will omit the $4 \mathrm{~d}$ factor of the zero mode in the following analysis, with the understanding that the internal spinors of even and odd chirality are tensored with even and odd $4 \mathrm{~d}$ spinors, respectively, which leads to a doubling of the number of solutions.

We define the Clifford vacuum as a state $|\Omega\rangle$ that satisfies the conditions

$$
\Gamma^{z}|\Omega\rangle=0, \quad \Gamma^{a}|\Omega\rangle=0 .
$$

All states of fixed chirality in Spin(10) will be divided into states of positive and negative charge with respect to the normal bundle $U(1)$. The states with positive chirality are

$$
\epsilon_{+}=\phi|\Omega\rangle+\phi_{\bar{a}} \Gamma^{\bar{a}}|\Omega\rangle+\phi_{\bar{a} \bar{b}} \Gamma^{\bar{a} \bar{b}}|\Omega\rangle .
$$

The states with negative chirality are

$$
\epsilon_{-}=\phi_{\bar{z}} \Gamma^{\bar{z}}|\Omega\rangle+\phi_{\overline{a z}} \Gamma^{\overline{a z}}|\Omega\rangle+\phi_{\overline{z a} \bar{b}} \Gamma^{\overline{z a} \bar{b}}|\Omega\rangle .
$$

Note that

$$
\tilde{G}_{(3)}|\Omega\rangle=i G_{(3)}|\Omega\rangle, \quad \quad \tilde{G}_{(3)} \Gamma^{\bar{a}}|\Omega\rangle=-i \bar{G}_{(3)} \Gamma^{\bar{a}}|\Omega\rangle .
$$

We want to consider the effect of a primitive $(2,1) 3$-form $G_{3}$. Let us here consider the simplest case when the value of the axion-dilaton field is fixed at a constant due to fluxes (note that in a generic F-theory background, the axion-dilaton field will vary over the internal manifold). The Dirac equations, obtained from (4.13) and rewritten in terms of (5.2) and (5.3) are

$$
\begin{gathered}
\partial_{\bar{a}} \phi+4 g^{\bar{b} c} \partial_{c} \phi_{\bar{b} \bar{a}}=0, \\
g^{\bar{b} a} \partial_{a} \phi_{\bar{b}}=0, \\
\partial_{[\bar{a}} \phi_{\bar{b}]}=0,
\end{gathered}
$$

and

$$
\begin{gathered}
\partial_{\bar{a}}^{A} \phi_{\bar{z}}+4 g^{\bar{b} c} \partial_{c}^{A} \phi_{\bar{b} \overline{a z}}-i 2 \bar{G}_{\overline{a z} b} \phi^{b}=0, \\
g^{\bar{a} b} \partial_{b}^{A} \phi_{\overline{a z}}+4 i G_{a b \bar{z}} \phi^{a b}=0
\end{gathered}
$$




$$
\partial_{[\bar{a}}^{A} \phi_{\bar{b}] \bar{z}}=0 .
$$

On forms which also have a $\bar{z}$ index, as in [6], we have a covariant derivative $\partial^{A} \equiv \partial+A$ rather than the straight derivative; $A$ is a connection on the line bundle $N=K$. It plays no explicit role in the following and we will leave it implicit from now on. The analysis now proceeds exactly as in [6]. First, in the absence of the flux, by acting with $\bar{\partial} \equiv\left(d z^{\bar{a}} \wedge\right) \bar{\partial}_{\bar{a}}$ and $\bar{\partial}^{\dagger} \equiv\left(g^{a \bar{b}} \iota \partial_{\bar{b}}\right) \partial_{a}{ }^{4}$ on the equations, we see that all forms must be harmonic. A simple application of Serre duality on the set (5.3) of negative $U(1)$ charge zero modes shows their number to be equal to that of positive $U(1)$ charge zero modes. We conclude that in the absence of fluxes and if the orientifold projection is not imposed, the index $\chi_{D 3}$ vanishes for brane instantons wrapping 4-cycles in Calabi-Yau manifolds. This is consistent with the fact that one does not expect the $\mathcal{N}=2$ supersymmetry ( 8 supercharges) of type IIB CY compactifications to be broken by instanton effects. Note that this differs from the CY 4-fold compactifications of M-theory, which can experience superpotential generation due to instantons in agreement with the fact that these compactifications only preserve 4 supercharges, i.e. $\mathcal{N}=1$ supersymmetry (from the perspective of the effective $4 d$ theory).

In the presence of flux, by acting with the harmonic projector $\mathcal{H}$ (introduced in [6]) on the two flux dependent equations (5.8) and (5.9), we conclude that they have solutions only if the following conditions are satisfied

$$
\begin{aligned}
\mathcal{H}\left(\bar{G}_{\overline{a z} b} \phi^{b}\right) & =0, \\
\mathcal{H}\left(G_{a b \bar{z}} \phi^{a b}\right) & =0 .
\end{aligned}
$$

If solutions for $\phi^{b}$ or $\phi^{a b}$ exist, then the relation

$$
(1-\mathcal{H})(\omega)=\bar{\partial}\left(\bar{\partial}^{\dagger} G \omega\right)+\bar{\partial}^{\dagger}(\bar{\partial} G \omega)
$$

with $\omega=2 i \bar{G}_{\overline{a z} b} \phi^{b} \bar{m}$ or $\omega=-4 i G_{a b \bar{z}} \phi^{a b}$ and $G$ being the Green operator, can be used to solve, respectively, the equations (5.8) and (5.9). If we denote solutions to the Dirac equation by the tuple $\left(\phi, \phi_{\bar{a} \bar{b}}, \phi_{\bar{a} \bar{z}}, \phi_{\bar{a}}, \phi_{\bar{z}}, \phi_{\bar{a} \bar{b} \bar{z}}\right)^{T}$, then the space of solutions is spanned by the set

$$
\left\{\left(\begin{array}{c}
\phi^{\text {harm }} \\
0 \\
0 \\
0 \\
0 \\
0
\end{array}\right),\left(\begin{array}{c}
0 \\
0 \\
\phi_{\overline{a z}}^{\text {harm }} \\
0 \\
0 \\
0
\end{array}\right), \quad\left(\begin{array}{c}
0 \\
0 \\
0 \\
0 \\
\phi_{\bar{z} \text { harm }} \\
0
\end{array}\right),\left(\begin{array}{c}
0 \\
0 \\
0 \\
0 \\
0 \\
\phi_{\bar{a} \bar{b} \bar{z}}^{\text {harm }}
\end{array}\right), \quad\left(\begin{array}{c}
0 \\
0 \\
0 \\
\tilde{\phi}_{\bar{a}} \\
g^{\bar{a} b} \partial_{b}\left(G \omega_{\overline{a z}}\right) \\
\frac{1}{4} \bar{\partial}_{\bar{a}}\left(G \omega_{\bar{b}] \bar{z}}\right)
\end{array}\right), \quad\left(\begin{array}{c}
0 \\
\tilde{\phi}_{\bar{a} \bar{b}} \\
\bar{\partial}_{\bar{a}}\left(G \omega_{\bar{z}}\right) \\
0 \\
0 \\
0
\end{array}\right),\right.
$$

\footnotetext{
${ }^{4}$ Here $\iota_{\partial_{\bar{b}}}$ is the contraction with the vector $\partial_{\bar{b}}$ : it acts as $\iota_{\partial_{\bar{b}}} d \bar{z}^{\bar{a}_{1}} \wedge \ldots d \bar{z}^{\bar{a}_{p}}=p \delta_{\bar{b}}{ }^{\left[\bar{a}_{1}\right.} d \bar{z}^{\bar{a}_{2}} \wedge \ldots \wedge d \bar{z}^{\left.\bar{a}_{p}\right]}$. The covariant derivative can be replaced with straight derivatives here because for a Kähler manifold the Levi-Civita connection is non-vanishing only when all the coefficients are of the same type, as in $\Gamma_{\bar{b} \bar{c}}^{\bar{a}}$. From $\bar{\partial}$, it drops because of antisymmetrization.
} 
where $\omega_{\overline{a z}}=2 i \bar{G}_{\overline{a z b}} \tilde{\phi}^{b}$ and $\omega_{\bar{z}}=-4 i G_{a b \bar{z}} \tilde{\phi}^{a b} . \tilde{\phi}_{\bar{b}}$ and $\tilde{\phi}_{\bar{a} \bar{b}}$ are harmonic forms which in addition satisfy (5.11) and (5.12) respectively. The F-chirality weight of these zero modes, in the order presented in equation (5.14), is $(+---++)$.

\subsection{Orientifolding and the choice of gauge for fixing $\kappa$-symmetry}

To obtain the Dirac equation in gauge fixed form in section 4, we imposed the gauge (4.3). Before imposing the orientifold projection, we must ensure that this choice of gauge is compatible with the constraint on physical modes which follows from the orientifold projection.

The orientifold action in general is given by (see e.g. [27])

$$
\mathcal{O}=(-1)^{F_{L}} \Omega_{P} \sigma^{*}, \quad \mathcal{O}^{2}=1
$$

where $\Omega_{P}$ is worldsheet parity and $\sigma$ is an involution on the CY manifold. On fermions, $(-1)^{F_{L}} \Omega_{P}$ acts by exchanging the $R-N S$ and $N S-R$ sector, and in addition multiplying the $N S-R$ sector by -1 . Acting on the fermion doublet introduced above, this operator is represented by $-i \sigma_{2}$, with $\sigma_{i}$ being the usual Pauli matrices. It is useful to compare it with 28. where the transformations of all the fields are given. Namely, the operation $(-1)^{F_{L}} \Omega_{P}$ in (5.15]) consists of a combination of two symmetries in [28, one with $\pm \sigma_{3}$ acting on fermions and another one with $\pm \sigma_{1}$, thus giving $-\sigma_{3} \times \sigma_{1}=-i \sigma_{2}$. We should distinguish between two types of brane instanton configurations. If the worldvolume of the brane is not left invariant by $\sigma$, this action sends fermions on the brane to fermions on its mirror on the other side of the orientifold plane. In this case, the orientifold action is taken into account simply by disregarding the mirror brane. If, on the contrary, the worldvolume is sent to itself by $\sigma$, we have an action $\sigma^{*}$ on the worldvolume fermions. This translates into a constraint satisfied by the modes that are not projected out by the orientifold action, of the general form

$$
\left(1-\Gamma_{\mathcal{O}}\right) \theta=0 .
$$

Now, we need to fix a gauge for the $\kappa$-symmetry that is compatible with the orientifolding action. Fixing $\kappa$-symmetry gives rise to a constraint,

$$
\left(1-\Gamma_{\kappa}\right), \theta=0,
$$

with $\Gamma_{\kappa}$ depending on the choice of gauge. These two constraints can be simultaneously imposed if the projectors commute, i.e.

$$
\left[\Gamma_{\mathcal{O}}, \Gamma_{\kappa}\right]=0 \text {. }
$$

In the following section, we will study a number of examples. In all cases, we will find that the duality covariant gauge chosen in (4.3) is compatible with the orientifolding.

\section{Examples}

We now determine the index in some examples which have an M-theory lift, and verify that both the M-theory and the IIB analysis yield the same conclusion with regard to the possibility of generating a superpotential by brane instantons. 


\subsection{The $K 3 \times T^{2} / \mathbb{Z}_{2}$ orientifold}

The $K 3 \times \frac{T^{2}}{\mathbb{Z}_{2}}$ orientifold can be obtained as a limit of $\mathrm{F}$-theory on $K 3 \times K 3$. It is possible to turn on a flux that stabilizes the 16 D7 branes which are required for tadpole cancellation on top of the orientifold planes [5]. We will assume that this choice has been made, so that our analysis in the previous section, which was performed with a constant axion-dilaton, is applicable.

On the covering space $K 3 \times T^{2}$ of the orientifold, we will use $1, \ldots, 6$ to denote real internal directions and $1, \overline{1}, \ldots, 3, \overline{3}$ to denote complex internal directions, where the first 4 coordinates are in the $K 3$ direction, and the last 2 are in $T^{2}$. The involution $\sigma$ in our example acts on both directions $\left(x^{5}, x^{6}\right)$ of the $T^{2}$ as reflection, i.e. $\sigma:\left(x^{5}, x^{6}\right) \rightarrow\left(-x^{5},-x^{6}\right)$.

We will consider three types of D3 brane instanton configurations: on top of an O7 plane, parallel to it, or intersecting it along one complex dimension.

- We first consider the case when the D3 brane is on top of an O7 plane, i.e. is wrapping the $K 3$, and is at a fixed point of the orientifold action in the $T^{2}$ directions. In terms of the local coordinates introduced above, this implies that the world volume directions $a, b$ coincide with the 1,2 direction, while the normal direction $z$ is in the 3 direction.

Let us look at the action of $\sigma$ on the fermions. The reflection in the 5 and 6 direction is represented on spinors by $\sigma^{*} \epsilon(x)=\gamma^{56} \epsilon(\sigma(x))$. But, due to the results of the previous section, we are only interested in harmonic forms. In the current example, it so happens that the coefficients are always even under $\sigma$, hence we can retain only the multiplication by $\gamma^{56}$. Since we are mapping fermions to forms on the brane via

$$
\phi_{\bar{a}_{1} \ldots \bar{a}_{n}} d \bar{z}^{\bar{a}_{1}} \wedge \ldots \wedge d \bar{z}^{\bar{a}_{n}} \leftrightarrow \phi_{\bar{a}_{1} \ldots \bar{a}_{n}} \gamma^{\bar{a}_{1}} \ldots \gamma^{\bar{a}_{n}}|\Omega\rangle
$$

we can also read off the action of $\sigma^{*}$ on fermions by the action of the pullback on forms: for branes wrapping the $K 3$ and coincident with one of the orientifold planes, we see that $\sigma^{*}$ acts with a sign on sections of the normal bundle. This of course coincides with the action given by multiplication of spinors by $\gamma^{56}$.

As explained in subsection (5.2), we need to fix a gauge for the $\kappa$ symmetry that is compatible with the orientifolding action. We first check that the simple gauge $\theta_{2}=0$ is not compatible with orientifolding in our model. The gauge-fixing condition $\theta_{2}=0$ can also be written in the form

$$
\left(1-\sigma_{3}\right) \theta=0
$$

The orientifold projection would require that

$$
\left(1-i \sigma_{2} \sigma^{*}\right) \theta=0
$$

Thus we have two projectors $\left(1-\Gamma_{\kappa}\right)$ and $\left(1-\Gamma_{O 7}\right)$, with $\Gamma_{\kappa}=\sigma_{3}$ and $\Gamma_{O 7}=i \sigma_{2} \sigma^{*}$, where $\sigma^{*}$ commutes with $\sigma_{2}$. They are anticommuting, and therefore incompatible. 
Next, we consider the gauge-fixing condition for the spinor on the brane which we used in Section 3 to derive the Dirac operator (see eqs. (4.3), (4.7)-(4.9)), namely

$$
\left(1-\sigma_{2} \gamma^{5}\right) \theta=0
$$

where $\gamma^{5}$ is the product of 4 gamma-matrices on the brane. In the case of the brane wrapping the K3 that we are considering, these directions are 1234. The gauge fixing condition is hence explicitly given by

$$
\left(1-\sigma_{2} \gamma^{1234}\right) \theta=0
$$

Now $\Gamma_{\kappa}=\sigma_{2} \gamma^{1234}$ and $\Gamma_{O 7}=i \sigma_{2} \sigma^{*}$, where $\left[\sigma^{*}, \gamma^{1234}\right]=\left[\gamma^{56}, \gamma^{1234}\right]=0$. These two projectors commute. Hence, this gauge is consistent with orientifolding.

To perform our zero mode count, it is convenient to rewrite $\Gamma_{O 7}$ and $\Gamma_{\kappa}$ in terms of local complex coordinates,

$$
\begin{aligned}
& \left(1-\Gamma_{\kappa}\right) \theta=\left(1-\sigma_{2} \gamma^{1 \overline{1} 2 \overline{2}}\right) \theta=0 \\
& \left(1-\Gamma_{O 7}\right) \theta=\left(1-\sigma_{2} \gamma^{3 \overline{3}}\right) \theta=0 .
\end{aligned}
$$

Together these conditions imply that

$$
\left(1-\gamma^{1 \overline{1} 2 \overline{2} 3 \overline{3}}\right) \theta=0
$$

which means that both $\theta_{1}$ and $\theta_{2}$ are positive chirality spinors in the 6-dimensional compact space and due to chirality in $10 \mathrm{~d}$ they are also chiral in $R^{4}$. The zero modes with positive $U(1)$ charge, that survive this projection are the $2 h^{0,0}$ modes $\phi|\Omega\rangle$, and the $2 h^{0,2}$ modes $\phi_{\bar{a} \bar{b}} \Gamma^{\bar{a} \bar{b}}|\Omega\rangle$. The modes with negative $U(1)$ charge are the $2 h^{0,1}$ modes $\phi_{\overline{a z}} \Gamma^{\overline{a z}}|\Omega\rangle$. Since the D3 brane is wrapping K3 in this example, which has $h^{0,1}=0$, the latter modes are absent. Thus without flux contribution the result for the index is

$$
\frac{1}{2}\left(N_{+}-N_{-}\right)=\left(h^{0,0}+h^{0,2}\right)=2 .
$$

This is in agreement with the index derived in the M-theory lift of this setup. In M-theory we are using the divisor $D$ which is a product of $K 3_{1}$ and a $\mathbb{P}^{1}$ in a singular elliptical fiber of $K 3_{2}$. The holomorphic characteristic of this divisor is $\chi_{D}=\chi_{K 3 \times \mathbb{P} 1}=$ $\chi_{K 3}=2$. This hence excludes the possibility of a flux induced superpotential.

We now add a three-form flux which preserves half of the supercharges and fixes the complex moduli in such a way that the D7s are on top of the O7s. This has been shown to be possible in [5] in the M-theory picture. In the IIB picture, at this point in the moduli space the flux has the form

$$
G_{3}=c \Omega \wedge d \bar{z}
$$


where $c$ is a constant and $\Omega$ is the holomorphic 2 -form on $K 3$ at this point. ${ }^{5}$. We can now see that the condition

$$
\mathcal{H}\left(G_{a b \bar{z}} \phi^{a b}\right)=0
$$

forces $\phi_{\bar{a} \bar{b}}=0$, for the following reason. The form $\phi^{a b}$ on K3 is proportional to $\bar{\Omega}$. Thus we have to contract the flux with $\bar{\Omega}$ and we are left with just a harmonic form, so $\mathcal{H}$ acts trivially on it. This cuts $2 h^{0,2}$ zero modes from the spectrum, changing the index to

$$
\chi_{D 3}(G)=h^{0,0}=1 .
$$

Again, this result reproduces the M-theory analysis in the presence of a primitive $(2,2)$ flux, and allows us to conclude that instanton corrections to the superpotential for the modulus which contains the volume of $K 3$ are now possible.

- When the brane is not on top of one of the orientifold planes but only parallel, there are a few changes. First of all, even though the gauge-fixing condition is still (6.6), the orientifold action can be disregarded, since it relates fermions on the brane to fermions on a mirror brane. Hence, in the absence of fluxes no modes are cut, and we are left with $2 \times\left(h^{0,0}+h^{0,1}+h^{0,2}\right)$ modes both with positive and negative sign. This gives the index $\chi_{D 3}=0$. This is again consistent with the M-theory dual: the elliptic fibration reduces on the D3 brane to a product, and the M5 dual is thus $K 3 \times T^{2}$, which has vanishing holomorphic characteristic. Finally, when we add the flux, the relevant equation is again (6.11), since in this case there are no $\phi_{\bar{a}}$, as $h^{0,1}(K 3)=0$; as above, the flux is covariantly constant, so the harmonic projector $\mathcal{H}$ acts as the identity and all the $\phi_{\bar{a} \bar{b}}$ are cut. However, this time we still have the modes $\phi_{\bar{z}}$ and $\phi_{\bar{a} \bar{b} \bar{z}}$. The index then becomes

$$
\frac{1}{2}\left(N_{+}-N_{-}\right)=\left(h^{0,0}-h^{0,0}-h^{0,2}\right)=-1
$$

which is not compatible with superpotential generation.

- We finally consider a D3 brane wrapping a $\mathbb{P}^{1}$ in the $\mathrm{K} 3$ and the $T^{2}$. In this case, the worldvolume of the D3 brane intersects an O7 locus along one complex dimension. The worldvolume directions $a, b$ are now along 2,3 , while the normal direction $z$ is along 1 .

By (6.1), the action of $\sigma^{*}$ on spinors living on branes wrapping $T^{2}$ is governed by the action of $\sigma^{*}$ on $H\left(T^{2}\right)$. Clearly, elements of $H^{0}\left(T^{2}\right)$ and $H^{2}\left(T^{2}\right)$ are even under this action, while elements of $H^{1}\left(T^{2}\right)$ are odd, thus reproducing once again that $\sigma^{*}$ acts on spinors via multiplication by $\gamma^{56}$. The conclusion reached above that the $\kappa$-symmetry gauge $\theta_{2}=0$ is not compatible with orientifolding hence applies here as well. The duality covariant gauge for the present brane instanton configuration reads

$$
\left(1-\sigma_{2} \gamma^{1256}\right) \theta=0 .
$$

With $\Gamma_{\kappa}=\sigma_{2} \gamma^{1256}$ and $\Gamma_{O 7}=i \sigma_{2} \sigma^{*}$ and $\left[\sigma^{*}, \gamma^{1256}\right]=\left[\gamma^{56}, \gamma^{1256}\right]=0$, we conclude that the two projectors commute. We see that the duality covariant gauge is compatible with orientifolding for this configuration as well.

\footnotetext{
${ }^{5}$ This choice of flux also stabilizes the axion-dilaton and other moduli in this model $10,11$.
} 
The gauge-fixing condition on the brane here reads

$$
\left(1-\sigma_{2} \gamma^{2 \overline{2} 3 \overline{3}}\right) \theta=0 .
$$

The orientifold condition requires

$$
\left(1-\sigma_{2} \gamma^{3 \overline{3}}\right) \theta=0 .
$$

Together these conditions imply that

$$
\left(1-\gamma^{2 \overline{2}}\right) \theta=0 .
$$

The zero modes that survive this projection are the $2 h^{0,0}\left(\mathbb{P}^{1} \times T^{2}\right)=2$ states from $\phi|\Omega\rangle$ and the $2 h^{0,1}\left(T^{2}\right)=2$ states from $\phi_{\overline{3}}|\Omega\rangle$ of a positive $U(1)$ charge, as well as $2 h^{0,2}\left(\mathbb{P}^{1} \times T^{2}\right)=0$ modes from $\phi_{\bar{z}}|\Omega\rangle$ and $2 h^{0,1}\left(\mathbb{P}^{1}\right)=0$ modes from $\phi_{\overline{3} \bar{z}}|\Omega\rangle$ of a negative $U(1)$ charge, where we have used Serre duality to count the number of the negative $U(1)$ charge modes. As a result, in the absence of fluxes the index is

$$
\frac{1}{2}\left(N_{+}-N_{-}\right)=2 .
$$

Note that the M-theory lift of this divisor is the same as in the previous example, $D=K 3 \times \mathbb{P}^{1}$, and $\chi_{D}=2$.

In the presence of flux, the 1 -form $\phi_{\overline{3}}$ must satisfy the additional constraint (5.11)

$$
\mathcal{H}\left(\bar{G}_{\overline{12} 3} \phi^{3}\right)=0 .
$$

Since the argument of the projector is a multiple of the $(0,2)$ form of the K3, the only solution of this constraint is $\phi_{\overline{3}}=0$. Thus, when the flux is turned on only the two zero modes from $\phi|\Omega\rangle$ survive yielding the index $\chi=1$. As in the M-theory analysis, the conclusion is that in the presence of the flux the instantons can generate a superpotential also in this case.

\subsection{D3-branes on general Fano manifolds}

We can now generalize the $K 3 \times \frac{T^{2}}{\mathbb{Z}_{2}}$ example, by considering the base manifold of the elliptically fibered F-theory fourfold to be an arbitrary Fano manifold. ${ }^{6}$

A general elliptic fibration is described by the usual Weierstrass equation describing an elliptic curve, $y^{2}=x^{3}+f x+g$, but where $f$ and $g$ are let vary over a base manifold $B$. For consistency of the equation, it turns out that $f$ and $g$ must be sections of $\mathcal{L}^{4}$ and $\mathcal{L}^{6}$ respectively, where $\mathcal{L}$ is a line bundle over $B$. If we want the total space of the fibration to be a Calabi-Yau, we have to take $\mathcal{L}=K^{-1}$, the inverse of the canonical line bundle of $B$. The axion-dilaton is, in general, determined by $f$ and $g$ through $j(\tau)=\frac{24}{1+\left(3^{3} g^{2}\right) /\left(2^{2} f^{3}\right)}$, where

\footnotetext{
${ }^{6}$ By definition, Fano manifolds have positive anticanonical bundle. This condition will come into play below equation (6.20).
} 
$j(\tau)$ is a certain known function. In general, $\tau$ will vary and will be outside perturbative control over some region of the base; but we can also make it to be constant if we choose $f$ and $g$ appropriately. Since $\tau$ only depends on the ratio $g^{2} / f^{3}$, if this ratio is constant so is $\tau$. This can be solved by taking

$$
f=a q^{2}, \quad g=b q^{3},
$$

with $a$ and $b$ being some constants and $q$ being a section of $K^{-2}$. If $B$ is a Fano manifold, by definition $K^{-1}$ is positive and hence has holomorphic sections, as does $K^{-2}$. We will call $C$ the zero locus of $q$.

All this is essentially a generalization of the example $B=\frac{T^{2}}{\mathbb{Z}_{2}}=\mathbb{P}^{1}$ considered in 34] and (taking the product with a spectator $K 3$ ) in section 6.1. There, $K^{-1}$ has degree 2, so $K^{-2}$ has degree 4 . Thus $q$ has 4 zeros. The monodromy argument of 34 tells us that on each of these 4 zeros there is an O7 and four D7s. This is consistent with the fact that the axion-dilaton is constant, since the tadpole is canceled locally. The monodromy argument is local and also goes through for a general Fano $B$, going around the zero locus of $q$. So on this zero locus there is an O7 with $4 \mathrm{D} 7 \mathrm{~s}$, once again. The manifold $B^{\prime}$ whose $\mathbb{Z}_{2}$ quotient gives $B$ (the generalization of $T^{2}$ in the $\mathbb{P}^{1}$ example above) can be described as in [35] as a double (branched) covering of $B$ by the equation $\xi^{2}=q$; the $B^{\prime}$ thus described is also a Calabi-Yau.

We now want to put a D3 on top of the zero locus of $q$ (or of one of its components, if it has more). The analysis of the first case in section 6.1 goes through, and we repeat it here. First of all, the gauge fixing and the orientifold projection look locally around the brane like (6.6) and (6.7). Then we can once again combine them to get a condition (6.8). This says that $\theta_{i}$ have positive internal chirality. This leaves us with only the modes $\phi, \phi_{\bar{a} \bar{b}}$ (to be counted with + sign) and $\phi_{\bar{a} \bar{z}}$, which can be dualized to a $\tilde{\phi}_{\bar{b}}$ (to be counted with a sign -). So in this case the index is

$$
\chi_{D 3}=h^{0,0}-h^{0,1}+h^{0,2},
$$

which just happens to be the holomorphic $\chi$ of the zero locus $C$.

Before turning on the flux, we can check that this index coincides with the one in Mtheory. The locus $C$ on which the D3 brane is sitting is exactly where the elliptic fibration gets singular. The blow-up of this singularity gives a chain of $\mathbb{P}^{1}$ 's intersecting in points. The dual of the D3 in M-theory is then wrapping one of these $\mathbb{P}^{1}$ along the fibre, and $C$ along the base. The holomorphic characteristic of this lift is then

$$
\chi_{M 5}=\chi\left(\mathbb{P}^{1}\right) \times \chi_{D 3}=\chi_{D 3}
$$

(since $\chi\left(\mathbb{P}^{1}\right)=1$ ).

When we add a flux, we are led again to consider the equations of section 5 . The only difference is that now some of the modes are not there because they have been projected away. We are now left with the sole condition $\mathcal{H}\left(G_{a b \bar{z}} \phi^{a b}\right)=0$, which determines whether or not the mode $\phi_{\bar{a} \bar{b}}$ is lifted. This of course has to be determined case by case, but we can once again check consistency with the M-theory picture. Indeed, the M-theory dual of $F$ and $H$ is $F_{\mathrm{M} \text {-theory }}=G \wedge d \bar{\zeta}$, where $\zeta$ is the holomorphic coordinate on the fibre, as follows 
from supersymmetry. Using this, the condition $\mathcal{H}\left(G_{a b \bar{z}} \phi^{a b}\right)=0$ is dual in M-theory to the condition

$$
\mathcal{H}\left(F_{a b \bar{c} \bar{z}} \phi^{a b}\right)=0
$$

which is just the condition obtained in [6].

As a final comment, we can also consider the case in which the D3 brane sits on a locus $D$ which never intersects $C$, generalizing the second brane instanton configuration of section 6.1. In that case, the orientifold projection only relates the D3 to a mirror D3, and it does not cut any of the modes. Thus the index is clearly zero. On the other hand, the elliptic fibration is never degenerating on $C$, since the D3 never intersects the degenerating locus $C$. So the dual M5 is a non-singular $T^{2}$ fibration over $C$, and its holomorphic characteristic gives $\chi_{M 5}=\chi\left(T^{2}\right) \times \chi_{D 3}=0$ (since $\left.\chi\left(T^{2}\right)=0\right)$. So the two indices agree once again.

One might wonder whether a generalization of the third brane instanton configuration of section 6.1 is also possible. One could consider a case in which the D3 brane locus $D$ has a non-zero intersection with the O7/D7 locus $C$. It is certainly possible to compute the holomorphic characteristic of the dual M5 (for example as in [4), but a general computation is much less clear in the type IIB setting. It would be interesting to explore this class of examples, and compare them with its M-theory dual.

\subsection{The case $T^{6} / \mathbb{Z}_{2}$}

Finally, we will briefly discuss an example with O3 rather than O7 orientifold planes, and see how it also fits into our analysis. Let us consider an orientifold defined by $\Omega_{P}(-1)^{F_{L}} \sigma^{*}$, where $\sigma$ is now the reflection of all the coordinates of $T^{6}$. This configuration involves 64 O3 planes. We can consider two types of D3 brane instanton configurations: a D3 brane on top of an $\mathrm{O} 3$ plane, or away from it. In the first case, the orientifold projection keeps only modes that satisfy the constraint

$$
\left(1-i \sigma_{2} \gamma^{x^{1} y^{1} x^{2} y^{2} x^{3} y^{3}}\right) \theta \equiv\left(1-\Gamma_{1}\right) \theta=0
$$

As in the $K 3 \times \frac{T^{2}}{\mathbb{Z}_{2}}$ example, there would also be an action of $\sigma$ on the argument of the spinor, but since we are dealing here with harmonic forms only (for which the coefficients are all constant in this case, and in particular even), we can disregard the argument altogether. On the other hand, when the D3 brane is not on top of one of the O3 planes, the worldvolume of the brane is sent to its mirror by $\sigma$, and the orientifold projection can be disregarded.

Now let's consider the question of choosing a gauge for $\kappa$-symmetry compatible with the constraint (6.24). $\theta_{2}=0$ is not compatible with this constraint: as before, we can write this choice of gauge as $\left(1-\sigma_{3}\right) \theta=0$; since $\Gamma_{1}$ involves $\sigma_{2}$, the constraint $\theta_{2}=0$ clearly does not commute with the orientifold constraint (6.24).

Next, we consider the duality covariant gauge-fixing condition for the spinor on the brane of the form

$$
\left(1-\sigma_{2} \gamma^{5}\right) \theta \equiv\left(1-\Gamma_{O 3}\right) \theta=0 .
$$

Recall that $\gamma^{5}$ is the product of the 4 gamma-matrices on the brane. Since $\left[\Gamma_{\kappa}, \Gamma_{O 3}\right]=0$, this gauge is once again the one that is consistent with orientifolding. 
Together, (6.25) and (6.24) give

$$
\left(1-\gamma^{z \bar{z}}\right) \theta=0 .
$$

This cuts all states in $\epsilon_{-}$. The total number of zero modes is now only $2 \times\left(h^{0,0}+h^{0,1}+h^{0,2}\right)=$ $2(1+2+1)=8$ and the index is equal to 4 .

We can compare this 4 with the M-theory dual $T^{8} / \mathbb{Z}_{2}\left[36\right.$. The dual M5 wraps a $T^{2} / \mathbb{Z}_{2}$ locus. The holomorphic characteristic of this orbifold is simply $h^{0,0}+h^{2,0}=4$ (since odd cohomology is projected out by the $\mathbb{Z}_{2}$ action).

Let us now add the flux. For the model of $\frac{T^{6}}{\mathbb{Z}_{2}}$ the flux which preserves $\mathcal{N}=1$ supersymmetry (but not larger) is of the form [37]

$$
G_{3} \sim d z^{1} \wedge d z^{2} \wedge d \bar{z}^{3}+d z^{2} \wedge d z^{3} \wedge d \bar{z}^{1}+d z^{3} \wedge d z^{1} \wedge d \bar{z}^{2},
$$

where $z^{i}=x^{i}+i y^{i}$. This is once again covariantly constant, so in the conditions (5.11) and (5.12) the contraction of the modes $\phi_{\bar{a} \bar{b}}$ and $\phi_{\bar{a}}$ with $G$ is automatically harmonic. This means that $h^{0,1}$ and $h^{0,2}$ are cut. Hence we are left with $2 h^{0,0}=2$ positive chirality zero modes. The index equals 1 , and we have instanton corrections.

Finally, let us consider the case in which no orientifold plane meets the brane, a case which has been considered recently in [12]. The orientifold projector can now be disregarded. Before introducing the flux, we have the modes $\phi, \phi_{\bar{a}}, \phi_{\bar{a} \bar{b}}$ and $\phi_{\bar{z}}, \phi_{\bar{a} \bar{z}}, \phi_{\bar{a} \bar{b} \bar{z}}$, which exactly cancel each other, leaving the index 0 . In the presence of fluxes, the relevant equations are now (5.11) and (5.12). Since the flux (6.27) is covariantly constant, as in the cases above the harmonic projector $\mathcal{H}$ acts as the identity, and all the modes $\phi_{\bar{a}}$ and $\phi_{\bar{a} \bar{b}}$ are lifted. This leaves us with the index

$$
\chi_{D 3}=h^{0,0}-h^{0,0}-h^{0,1}-h^{0,2}=-2-1=-3
$$

which is incompatible with superpotential generation and agrees with the results of [12].

\section{Discussion}

In this paper we have demonstrated that the coupling of worldvolume fermions to fluxes opens up the possibility of having instanton corrections to the superpotential from Euclidean D3 branes in cases where such corrections were ruled out in the absence of flux. We have introduced an index for the Dirac operator on the D3 brane,

$$
\chi_{D 3}=\frac{1}{2}\left(N_{+}-N_{-}\right),
$$

where $N_{ \pm}$is the number of fermionic zero modes having a $U(1)$ charge $\pm \frac{1}{2}$ in the normal direction. We argue that $\frac{1}{2}\left(N_{+}-N_{-}\right)$must be equal to 1 to allow instanton corrections to the superpotential. In the presence of fluxes, the counting of the fermionic zero modes weighted with the $U(1)$ charge may be modified, compared to the cases without fluxes, as we have shown in some interesting examples. 
In particular, in type IIB string theory compactified on a $K 3 \times T^{2} / \mathbb{Z}_{2}$ orientifold, it is possible to have instanton corrections to the superpotential for the D3 brane wrapped on $K 3$ at the orientifold locus as well as on $\mathbb{P}^{1} \times T^{2} / \mathbb{Z}_{2}$ with $\mathbb{P}^{1}$ being a curve in the $K 3$. This is in agreement with the corresponding results in M-theory established in [6]. In the presence of such contributions to the superpotential, the stabilization of all moduli in type IIB string theory compactified on a $K 3 \times T^{2} / \mathbb{Z}_{2}$ orientifold is within reach, and in fact has been achieved from the perspective of M-theory compactified on $K 3 \times K 3$ in [30] and in its F-theory dual.

For cosmological applications, it is interesting to add D3 branes extended along the physical non-compact directions to the mix. In the tree-level analysis, the positions of the D7 branes are generically fixed by the flux, whereas the positions of the D3 branes remain unfixed moduli as long as no two-form along the D7 worldvolume is introduced. The D3 brane positions in $T^{2} / \mathbb{Z}_{2}$ belong to vector multiplets and the positions in $K 3$ belong to hypermultiplets. In the model of [9, 33] the distance between the D3 and D7 branes serves as the inflaton. This model has the attractive feature that instead of having to fine-tune the inflaton potential, its flatness arises due to a dynamical mechanism, namely the slightly broken shift symmetry [33, 9] in the inflaton direction due to a not-self-dual 2-form flux on the D7 branes. In the presence of 2-form flux on the D7s the cosmological system has 2 stages of evolution, one in a locally stable de Sitter valley with almost flat directions when the D3 is far from the D7s, and the second stage when the absolute minimum of the potential is reached, when both the D7s and the D3 are stabilized and the D-flatness condition is restored. In many recent studies of flux vacua this feature of cosmological D-term inflation model has not been appreciated yet and included in the analysis. Other features of the model include a well understood exit from the inflation stage with reheating and the value of the tilt of the spectrum $n_{s}=0.98$ which is the central value of the current data.

While Kähler moduli and D3 brane positions enter the action in different ways ${ }^{7}$, one can ask the question whether the latter are also lifted in the presence of brane instantons. For example, dependence on these positions could arise in the determinant prefactor, as argued in [31] in the absence of fluxes, and in [32, whose underlying assumption is that the duality between $K 3 \times K 3$ and type II on a Calabi-Yau is still valid in the presence of fluxes. In the related context of gaugino condensation giving rise to superpotentials, [39] argue for a dependence on D3 brane positions arising due to threshold corrections. More work is required to clarify the status of D3 brane positions in the context of the model presented in our paper, with instanton corrections rather than gaugino condensation giving rise to Kähler moduli dependence in the superpotential.

If the mobility of D3 branes in the de Sitter valley when far from the D7s survives instanton corrections, string cosmology will receive a significant boost. This will constitute the last step in deriving D-term inflation [41] with its various attractive phenomenological

\footnotetext{
${ }^{7}$ Kähler moduli are given by volumes of various 4 -cycles in the $K 3 \times T^{2} / \mathbb{Z}_{2}$ orientifold, and as such appear in the classical action of brane instantons. Instanton corrections to the superpotential hence give rise to a dependence of the form $\sim e^{-\rho}$, with $\rho$ indicating a generic Kähler modulus. In contrast, the positions of D3 branes enter more delicately in the theory, e.g. determining the masses of modes of strings stretching between D3 and D7 branes.
} 
features from string theory.

\section{Acknowledgments}

We are grateful to P. Aspinwall, M. Douglas, S. Kachru, A. Linde, D. Lüst, L. Martucci, F. Marchesano, J. McGreevy, S. Reffert, S. Stieberger, P. K. Tripathy and S. Trivedi for useful discussions. This work was supported by NSF grant 0244728. The work of AK was also supported by the U.S. Department of Energy under contract number DE-AC02-76SF00515. The work of E.B. and D. S. was supported by the EU MRTN-CT-2004-005104 grant 'Forces Universe' in which E.B. is associated to Utrecht University, and by the MIUR contract no. 2003023852 (D.S.).

\section{A Conventions and definitions}

We denote (Lorenzian) worldvolume indices by $\alpha=0,1,2,3$ and (Lorenzian) target space indices by $m=0,1,2, \cdots, 9$. The corresponding flat indices are underlined. The tendimensional chiral operator is $\Gamma_{11}=\Gamma_{\underline{0 \ldots 9}}$ with $\left(\Gamma_{11}\right)^{2}=1$.

The matrix $\Gamma_{D 3}$ of the kappa-symmetry projector is defined by

$$
\Gamma_{D 3}=e^{-a / 2} \sigma_{2} \gamma_{5} e^{+a / 2},
$$

with $a(\mathcal{F})$ some function of $\mathcal{F}\left[18\right.$ and $\gamma_{5}=i \gamma_{\underline{0123}}, \quad\left(\gamma_{5}\right)^{2}=1^{8}$. In the absence of worldvolume flux, i.e. $\mathcal{F}_{\alpha \beta}=0$, we have $a=0$ and the expression for $\Gamma_{D 3}$ reduces to

$$
\Gamma_{D 3}=\sigma_{2} \gamma_{5}
$$

The gravitino and dilatino supersymmetry rules are given by $\delta_{\epsilon} \psi_{m}=\delta \psi_{m} \epsilon$ and $\delta_{\epsilon} \lambda=\delta \lambda \epsilon$ where $\epsilon$ is a doublet of $10 \mathrm{~d}$ chiral spinors and

$$
\delta \psi_{m}=\delta \psi_{m}^{N S}+\delta \psi_{m}^{R R}, \quad \delta \lambda=\delta \lambda^{N S}+\delta \lambda^{R R}
$$

with

$$
\begin{aligned}
\delta \psi_{m}^{N S} & =\nabla_{m}+\frac{1}{4 \cdot 2 !} H_{m n p} \tilde{\Gamma}^{n p} \sigma_{3} \\
\delta \psi_{m}^{R R} & =\frac{1}{8} e^{\phi}\left[-F_{n} \tilde{\Gamma}^{n}\left(i \sigma_{2}\right)+\frac{1}{3 !} F_{n p q}^{\prime} \tilde{\Gamma}^{n p q} \sigma_{1}+\frac{1}{2 \cdot 5 !} F_{n p q r t} \tilde{\Gamma}^{n p q r t}\left(i \sigma_{2}\right)\right] \Gamma_{m} \\
\delta \lambda^{N S} & =\frac{1}{2}\left(\Gamma^{m} \partial_{m} \phi+\frac{1}{2 \cdot 3 !} H_{m n p} \Gamma^{m n p} \sigma_{3}\right) \\
\delta \lambda^{R R} & =-\frac{1}{2} e^{\phi}\left[-F_{m} \Gamma^{m}\left(i \sigma_{2}\right)+\frac{1}{2 \cdot 3 !} F_{m n p}^{\prime} \Gamma^{m n p} \sigma_{1}\right]
\end{aligned}
$$

\footnotetext{
${ }^{8}$ For the passage from Minkowski to Euclidean space it is important to remember that in Euclidean space $\gamma_{5}$ with $\left(\gamma_{5}\right)^{2}=1$ is defined as $\gamma_{5}=\gamma_{\underline{1234}}$ and hence is the same as $\gamma_{5}$ in Minkowski space since $\gamma_{\underline{4}}=-i \gamma_{\underline{0}}$.
} 
where $\nabla_{m}=\partial_{m}+\frac{1}{4} \Omega_{m} \underline{n p} \tilde{\Gamma}_{\underline{n p}}$ is the covariant derivative. The different curvatures appearing in the supersymmetry rules are defined by $H_{(3)}=d B_{(2)}, F_{(3)}=d C_{(2)}$ and

$$
F_{(1)}=d C_{(0)}, \quad F_{(3)}^{\prime}=F_{(3)}-C_{(0)} H_{(3)}, \quad F_{(5)}=d C_{(4)}+H_{(3)} \wedge C_{(2)} .
$$

Note that in eqs. A.4 and (A.5) we put tilde on antisymmetric combinations of $16 \times 16$ gamma-matrices to indicate that they carry the chiral spinor indices opposite to those in eqs. (4.5) and (4.6). This distinction is important because e.g. $\tilde{\Gamma}^{m_{1} \cdots m_{5}}$ are anti-self-dual while $\Gamma^{m_{1} \cdots m_{5}}$ are self-dual.

\section{References}

[1] O. DeWolfe, A. Giryavets, S. Kachru and W. Taylor, "Type IIA moduli stabilization," arXiv:hep-th/0505160.

[2] M. Davidse, F. Saueressig, U. Theis and S. Vandoren, "Membrane instantons and de Sitter vacua," arXiv:hep-th/0506097; F. Saueressig, U. Theis and S. Vandoren, "On de Sitter vacua in type IIA orientifold compactifications," arXiv:hep-th/0506181.

[3] E. Witten, "Non-Perturbative Superpotentials In String Theory," Nucl. Phys. B 474, 343 (1996) arXiv:hep-th/9604030;

[4] D. Robbins and S. Sethi, "A barren landscape," Phys. Rev. D 71, 046008 (2005) arXiv:hep-th/0405011;

[5] L. Gorlich, S. Kachru, P. K. Tripathy and S. P. Trivedi, "Gaugino condensation and nonperturbative superpotentials in flux compactifications," arXiv:hep-th/0407130.

[6] R. Kallosh, A. K. Kashani-Poor and A. Tomasiello, "Counting fermionic zero modes on M5 with fluxes," arXiv:hep-th/0503138.

[7] N. Saulina, "Topological constraints on stabilized flux vacua," arXiv:hep-th/0503125.

[8] R. Kallosh and D. Sorokin, "Dirac action on M5 and M2 branes with bulk fluxes," arXiv:hep-th/0501081.

[9] K. Dasgupta, C. Herdeiro, S. Hirano and R. Kallosh, "D3/D7 inflationary model and M-theory," Phys. Rev. D 65, 126002 (2002) arXiv:hep-th/0203019. F. Koyama, Y. Tachikawa and T. Watari, "Supergravity analysis of hybrid inflation model from D3-D7 system," Phys. Rev. D 69, 106001 (2004) [Erratum-ibid. D 70, 129907 (2004)] arXiv:hep-th/0311191.

[10] P. K. Tripathy and S. P. Trivedi, "Compactification with flux on K3 and tori," JHEP 0303, 028 (2003) arXiv:hep-th/0301139. 
[11] L. Andrianopoli, R. D'Auria, S. Ferrara and M. A. Lledo, "4-D gauged supergravity analysis of type IIB vacua on K3 x T**2/Z(2)," JHEP 0303, 044 (2003) arXiv:hep-th/0302174.

[12] P. K. Tripathy and S. P. Trivedi, "D3 brane action and fermion zero modes in presence of background flux," arXiv:hep-th/0503072.

[13] J. Gomis, F. Marchesano and D. Mateos, "An open string landscape," arXiv:hep-th/0506179.

[14] M. J. Duff, J. T. Liu and R. Minasian, "Eleven-dimensional origin of string / string duality: A one-loop test," Nucl. Phys. B 452, 261 (1995) arXiv:hep-th/9506126.

[15] E. Witten, "Five-branes and M-theory on an orbifold," Nucl. Phys. B 463, 383 (1996) arXiv:hep-th/9512219. "Five-brane effective action in M-theory," J. Geom. Phys. 22, 103 (1997) |arXiv:hep-th/9610234.

[16] D. Freed, J. A. Harvey, R. Minasian and G. W. Moore, "Gravitational anomaly cancellation for M-theory fivebranes," Adv. Theor. Math. Phys. 2, 601 (1998) arXiv:hep-th/9803205.

[17] K. Becker, M. Becker and A. Strominger, "Five-branes, membranes and nonperturbative string theory," Nucl. Phys. B 456 (1995) 130 arXiv:hep-th/9507158.

[18] E. Bergshoeff, R. Kallosh, T. Ortín, and G. Papadopoulos, "kappa-symmetry, supersymmetry and intersecting branes", Nucl. Phys. B502 (1997), 149; arXiv:hep-th/9705040.

[19] D. Marolf, L. Martucci and P. J. Silva, "Actions and fermionic symmetries for D-branes in bosonic backgrounds," JHEP 0307, 019 (2003) arXiv:hep-th/0306066; D. Marolf, L. Martucci and P. J. Silva, "Fermions, T-duality and effective actions for D-branes in bosonic backgrounds," JHEP 0304, 051 (2003) arXiv:hep-th/0303209.

[20] R. Kallosh, "Duality symmetric quantization of superstrings," Phys. Rev. D 52, 6020 (1995) arXiv:hep-th/9506113; R. Kallosh, "Quantization of p-branes, D-p-branes and M-branes," Nucl. Phys. Proc. Suppl. 68, 197 (1998) arXiv:hep-th/9709202.

[21] I. Bandos, P. Pasti, D. Sorokin, M. Tonin and D. Volkov, "Superstrings and supermembranes in the doubly supersymmetric geometrical approach," Nucl. Phys. B446, 79 (1995) arxiv:hep-th/9501113.

[22] I. Bandos, D. Sorokin and M. Tonin, "Generalized action principle and superfield equations of motion for D=10 D-p-branes", Nucl. Phys. B497, 275 (1997) arxiv:hep-th/9701127. 
[23] P. S. Howe and E. Sezgin, " $\mathrm{D}=11, \mathrm{P}=$ 5", Phys. Lett. B 394 (1997) 62 arXiv:hep-th/9611008.

P. S. Howe, E. Sezgin and P. C. West, "Covariant field equations of the M-theory five-brane," Phys. Lett. B 399, 49 (1997) arXiv:hep-th/9702008.

P. S. Howe, E. Sezgin and P. C. West, "Aspects of superembeddings," arXiv:hep-th/9705093.

[24] D. P. Sorokin, "Superbranes and superembeddings," Phys. Rept. 329, 1 (2000) arXiv:hep-th/9906142.

[25] L. Martucci, J. Rosseel, D. Van den Bleeken and A. Van Proeyen, "Dirac actions for D-branes on backgrounds with fluxes," arXiv:hep-th/0504041.

[26] M. Grana, "D3-brane action in a supergravity background: The fermionic story," Phys. Rev. D 66, 045014 (2002) arXiv:hep-th/0202118.

[27] A. Dabholkar, "Lectures on orientifolds and duality," arXiv:hep-th/9804208.

[28] E. Bergshoeff, M. de Roo, B. Janssen and T. Ortin, "The super D9-brane and its truncations," Nucl. Phys. B 550, 289 (1999) arXiv:hep-th/9901055.

[29] M. Grana and J. Polchinski, "Supersymmetric three-form flux perturbations on AdS(5)," Phys. Rev. D 63, 026001 (2001) arXiv:hep-th/0009211.

[30] P. S. Aspinwall and R. Kallosh, "Fixing all moduli for M-theory on K3 x K3," arXiv:hep-th/0506014.

[31] O. J. Ganor, Nucl. Phys. B 499, 55 (1997) arXiv:hep-th/9612077.

[32] P. Berglund and P. Mayr, "Non-perturbative superpotentials in F-theory and string duality," arXiv:hep-th/0504058.

[33] J. P. Hsu, R. Kallosh and S. Prokushkin, "On brane inflation with volume stabilization," JCAP 0312, 009 (2003) arXiv:hep-th/0311077. H. Firouzjahi and S. H. H. Tye, "Closer towards inflation in string theory," arXiv:hep-th/0312020. J. P. Hsu and R. Kallosh, "Volume stabilization and the origin of the inflaton shift symmetry in string theory," JHEP 0404, 042 (2004) arXiv:hep-th/0402047.

[34] A. Sen, "F-theory and Orientifolds," Nucl. Phys. B 475, 562 (1996) arXiv:hep-th/9605150.

[35] A. Sen, "Orientifold limit of F-theory vacua," Phys. Rev. D 55, 7345 (1997) arXiv:hep-th/9702165.

[36] A. Sen, "Orbifolds of M-Theory and String Theory," Mod. Phys. Lett. A 11, 1339 (1996) arXiv:hep-th/9603113. 
[37] S. Kachru, M. B. Schulz and S. Trivedi, "Moduli stabilization from fluxes in a simple IIB orientifold," JHEP 0310, 007 (2003) arXiv:hep-th/0201028.

[38] D. Lust, P. Mayr, S. Reffert and S. Stieberger, "F-theory flux, destabilization of orientifolds and soft terms on D7-branes," arXiv:hep-th/0501139.

[39] M. Berg, M. Haack and B. Kors, "On the moduli dependence of nonperturbative superpotentials in brane inflation," arXiv:hep-th/0409282 L. McAllister, "An inflaton mass problem in string inflation from threshold corrections to volume stabilization," arXiv:hep-th/0502001.

[40] D. Lust, S. Reffert, W. Schulgin and S. Stieberger, "Moduli stabilization in type IIB orientifolds. I: Orbifold limits," arXiv:hep-th/0506090.

[41] P. Binetruy and G. Dvali, "D-term inflation," Phys. Lett. B 388, 241 (1996) hep-ph/9606342; ; E. Halyo, "Hybrid inflation from supergravity D-terms," Phys. Lett. B 387, 43 (1996) hep-ph/9606423. 